\title{
Shape and texture analysis of liver cell nuclei in hepatomas by computer aided microscopy
}

\author{
ROGER JAGOE, CHRISTOPHER SOWTER, * GERARD SLAVIN* \\ From the Clinical Research Centre and Northwick Park Hospital, Harrow, Middlesex, HA1 $3 U \mathrm{~J}$
}

SUMMARY Nuclei from non-neoplastic hepatocytes and from hepatoma cells have been examined by image analysis techniques which measure size, shape, and texture. There are significant differences in these parameters between malignant and non-neoplastic cells. When values are obtained from multiple cells in a single biopsy, discriminant programs satisfactorily separate benign and malignant tissues. Techniques of image analysis offer an opportunity to quantify the nuclear changes associated with malignancy and may provide an objective basis for tumour grading systems.

Changes in cell nuclei are important in the assessment of neoplasia by pathologists. In routine practice they are assessed subjectively and are used not only in diagnosis but also as the basis of grading systems which may be extrapolated as a guide to therapy or prognosis. There is a need to quantify such changes and to verify their importance. Measurements of nuclear features have been made in uterine carcinoma,' malignant lymphoma, ${ }^{2}$ normal lymphoid tissue ${ }^{3}$ gastric carcinoma, ${ }^{4}$ and urothelial tumours. ${ }^{56}$ Most of these measurements have been related to nuclear size and shape, though texture analysis has been applied in carcinoma of the breast. $^{\text {? }}$

We have previously distinguished between liver cell nuclei from normal hepatocytes and those from hepatoma cells by means of nuclear size and shape. ${ }^{8}$ In this report we have extended our observations, considering further measurements of shape and also of texture in the assessment of nuclear changes in neoplasia. Moreover, in this study definition of the nuclear boundary has been made semiautomatically by edge tracing routines rather than by a simple drawing technique.

\section{Material and methods}

Liver biopsy material was obtained from the diagnostic files of Northwick Park Hospital and the Clinical Research Centre. Ten livers coded as "normal"

\section{Accepted for publication 12 March 1984}

*Present address: Department of Histopathology, St Bartholomew's Hospital Medical College, West Smithfield, London and 10 livers coded as "hepatoma" were examined. All the material had been fixed in formol saline and processed by standard procedures to produce $4 \mu \mathrm{m}$ paraffin sections stained by haematoxylin and eosin.

The sections were then examined using a Magiscan (Joyce-Loebl) Image Analysis System, in which a standard 625 line TV camera is used to scan the microscope image. This signal is then digitised to a $512 \times 512$ square matrix of grey values or pixels. Each pixel has a discrete value between 0 and 63 which represents the minimum and maximum light intensities. Each section was examined using a $\times 100$ oil immersion objective, giving a resolution of $0 \cdot 2$ $\mu \mathrm{m} /$ pixel. Maximum nuclear contrast was obtained using a Kodak green filter. The grey level images of the liver cell nuclei were then analysed.

One section was examined from each liver, and liver parenchymal cell nuclei were chosen at random in each section. A computer program selected randomly any one of 81 possible fields. The boundaries of up to five hepatocyte nuclei nearest to the centre of the field were selected until a total of 30 nuclei had been counted. The nuclei were detected by a semiautomatic edge tracking program in about $85 \%$ of non-neoplastic cells and in about $60 \%$ of hepatocytes from neoplastic biopsies. Those nuclear boundaries not detected by the edge tracking routine were partially or entirely drawn by the operator using a light pen. The nuclear tracking routine will be reported separately.

The magnification gave a monitor image of about 1000 square pixels for the smallest nucleus examined. This was large enough to reduce the error of area measurement in repeated manual tracing of a nuclear boundary to less than $5 \%$. 
The delineated boundary of each nucleus was enlarged automatically so that a small amount of cytoplasm was included as background to the nucleus. This was done by expanding the distance of each boundary point from the centre of gravity by a factor of $1 \cdot 2$. The grey level values of each pixel point in this enlarged area were recorded on disk files.

\section{MEASUREMENTS}

Thirty measurements or derived values of nuclear size, shape, and texture were made.

\section{Size measurement}

(1) SIZE. The area within each nuclear boundary was used as a measurement of size.

\section{Shape measurements}

Eleven shape measurements were made on the nuclear boundary data.

(2) P2A. The simplest and best known shape measurement P2A (perimeter squared divided by $4 \times \mathrm{PI}$ $\times$ AREA) has been used by many workers and is a component of commercial packages. Deviations of the value from unity reflect deviations from circularity.

While P2A was computed from the original trace of the nuclear boundary other shape measurements are size dependent. To compensate for this the boundary of each nucleus was expanded about its centre of gravity to enclose an almost constant area. A smoothing procedure was applied to even out small irregularities generated by the normalisation. Ninety three percent of the resulting boundaries had areas within $0.5 \%$ of 10000 square pixels and the remainder were within $1 \%$ of this value. Ten measurements were derived from the normalised boundary.

(3) PERIM. The length of the normalised boundary.

Shape measurements were made based on alteration of a discrete boundary curvature function $\mathrm{f}(\mathrm{C})$. This is derived by applying a filtering technique to a direction coded boundary list. This consists of a sequence of integer values which represent changes in direction from point to point along the boundary. Left turn is coded as -1 , straight ahead as 0 , right turn as +1 . The filter acts by replacing each value in this list by the average of itself and its eight neighbours. This is repeated four times. ${ }^{10} \mathrm{~A}$ plot of the resulting function shows peaks and troughs corresponding to convexities and concavities along the boundary.

(4) AVCURV. Mean value of $f(C)$.

(5) STDCURV. Standard deviation of $f(C)$.

(6) MINCURV. Minimum value of $f(C)$.
(7) MAXCURV. Maximum value of $f(C)$.

(8) SSQCURV. Sum of squared values of $f(C)$.

Changes in the shape of the nuclear boundary were also measured by alterations in the distance between the boundary and the centre of gravity of the nucleus. Four values were obtained:

(9) AVDIS. The average distance of the nuclear boundary from the centre of gravity of the nucleus. (10) STDIS. The standard deviation of the distance of the nuclear boundary from the centre of gravity.

(11) MINDIS. The minimum distance of the nuclear boundary from the centre of gravity.

(12) MAXDIS. The maximum distance of the nuclear boundary from the centre of gravity.

\section{Texture and densitometric measurements}

Four categories of nuclear texture measurement were used.

(a) Simple grey level values and gradient statistics. These are derived from the grey level value of each pixel point in the nuclear image. The gradient strength, which measures the local rate of change in grey levels, was computed from the $3 \times 3$ Sobel operator (Fig. 1) for those operators where all the nine points were contained within the nuclear boundary. Six values were derived from these measurements:

(13) MGREY. Mean nuclear grey level over the whole nucleus.

(14) STDGREY. Standard deviation of the nuclear

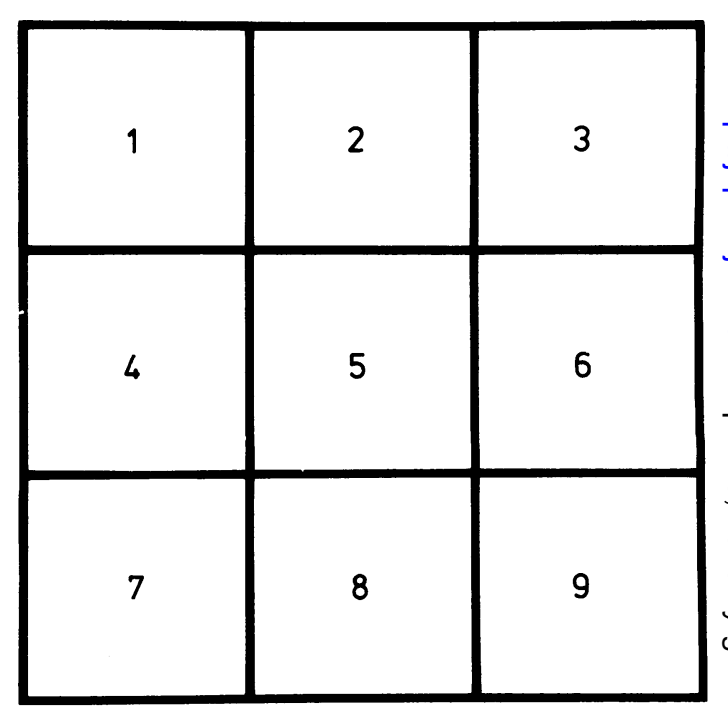

Fig. 1 Gradient values calculated from a $3 \times 3$ operator. If $G_{i}$ is the grey value of each point $(1 \leqslant i \leqslant 9)$ in the $3 \times 3$ area then the gradient strength at the central point (5) of the area is computed as: $G R A D=S Q R T\left(\left(G_{1}+2 \times G_{2}+G_{3}\right.\right.$ $\left.-G_{7}-2 \times G_{8}-G_{4}\right)^{2}+\left(G_{3}+2 \times G_{6}+G_{4}-G_{1}-2 \times G_{4}\right.$ $\left.-G_{7}\right)^{2}$ ). 
grey levels.

(15) STDGREY/MGREY. Coefficient of variation of nuclear grey levels.

(16) MGRAD. Mean gradient of nuclear grey level.

(17) STDGRAD. Standard deviation of the gradient of nuclear grey levels.

(18) STDGRAD/MGRAD. Coefficient of variation of the gradient of nuclear grey levels.

(19) IOD. The integrated nuclear optical density was estimated by computing

$$
\text { IOD }=-\Sigma\left(\log \left(G_{i j} / M G C\right)\right)
$$

where $G_{i j}$ is the grey level of a nuclear pixel at point $(i, j)$ and MGC is the mean grey level of the background cytoplasm.

(b) Measurements designed to reflect the distributions of grey levels relative to their distances from the nuclear boundary or relative to their angular distributions within the nucleus. These were undertaken because visual assessment suggested that neoplastic nuclei have a relatively higher concentration of densely stained material closer to the nuclear boundary than non-neoplastic nuclei.

(20) ARANGE. The annular range of grey levels was computed for each nucleus by calculating the range between the mean grey levels of eight bands at a relative distance $\operatorname{Dr}(0 \leqslant \mathrm{Dr} \leqslant 7)$ from the nuclear boundary. The relative distance $\mathrm{Dr}$ is related to the actual distance $\mathrm{Da}$ by the relation

$$
\mathrm{Dr}=\text { integral part }((8 \times \mathrm{Da}) / \mathrm{Dmax})
$$

where Dmax is the maximum internal distance from the boundary (Fig. 2).

(21) SRANGE. The sector range of grey levels is the range between the mean grey levels of eight equiangular sectors with the origin at the centre of gravity.

(22) MGREYO/MGREY. The ratio of the mean grey level of pixels in the outer most band to the mean grey level of the total nucleus.

(23) BMOM. A grey level moment about the original boundary was computed for all eight relative distances $(\mathrm{d}=\mathrm{Dr})$

$$
\text { BMOM }=\frac{\sum_{d=0}^{7}(d+1) \sum_{i=1}^{N_{d}} G_{i d}}{M G R E Y \times \sum_{d=0}^{7}(d+1) N_{d}}
$$

where $G_{i d}$ is the grey level of a point $i$ in band $d, N_{d}$ is the number of pixels in band d, and MGREY is the mean nuclear grey level over the whole nucleus.

In nuclei with evenly spread grey level values BMOM is close to unity. If hyperchromatic regions of the nucleus are represented by relatively lower

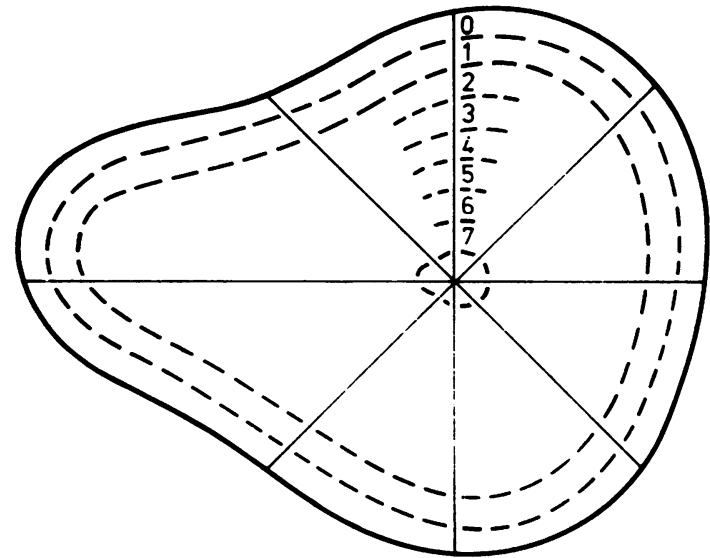

Fig. 2 Division of nuclear area into eight bands and eight sectors to assess the distribution of densely stained material in relation to the nuclear boundary.

grey values a nucleus with a darkly stained rim region will give a value greater than unity.

(c) Measurements extracted from co-occurrence or grey level spatial dependency matrices. These have been extensively reviewed in the published work ${ }^{\prime \prime}$ and used as discriminators of terrain types in Landsat images. ${ }^{12}{ }^{13}$ Only an outline of them is given here.

A nuclear image point has eight nearest neighbours in the directions $0,45,90, \ldots 360$ degrees. In each of these directions eight grey level density probability functions can be estimated for any distance d between image points. Each grey level density function can be written in the form:

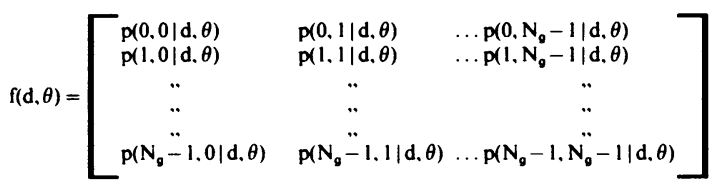

where $\mathrm{Ng}=$ the number of grey levels in the image $(0$ to $\mathrm{Ng}-1)$ and $\mathrm{p}(\mathrm{i}, \mathrm{j} \mid \mathrm{d}, \theta)$ is the estimated probability of a transition from grey level $i$ to grey level $j$ at a sampling interval $d$ in the direction $\theta$. The density functions for opposite directions are summed to give four symmetric matrices.

A digitised image may have 64 grey levels, but typically a nuclear region exhibits a range of only 15 to 30 grey levels. This range may then be divided into $\mathrm{N}$ intervals each of equal probability of occurrence. A transformed digital image is derived from the original by assigning to each pixel a value of 1 to $\mathrm{N}$, corresponding to the interval in which it lay in the original image. Thus the transformed image will have $\mathbf{N}$ possible grey levels and the probability mat- 
rices will be of size $\mathbf{N} \times \mathbf{N}$. This process is invariant with respect to monotonic grey level transformations such as alterations in light levels. ${ }^{9}$

In preliminary trials it was found that the power of the features derived from such matrices to discriminate normal from neoplastic nuclei did not vary significantly for different values of $\mathrm{N}$ above the value of $N=8$. In this study a value of $N=8$ was chosen. The raw digital data were preprocessed by the above method before the computation of the co-occurrence matrices. Since directional texture information cannot be relevant to randomly oriented cell nuclei, the four directional symmetric matrices were averaged to a single matrix. As unit sample interval between pixels was used $(d=1)$, the averaging is equivalent to tabulating the transitions of transformed grey levels between a central pixel and its eight surrounding neighbours. An example of matrices derived from Fig. 3 where the image has three grey levels $(0-2)$ is given in Table 1 .

Five commonly used texture features ${ }^{11}$ were computed from the co-occurrence matrices for each nucleus.

(24) ENTROPY. This is calculated as:

$$
E N T=-\sum_{i=0}^{N_{g}-1} \sum_{j=0}^{N_{g}-1} p(i, j) \ln (p(i, j))
$$

where $N_{g}$ is the number of equiprobable grey level intervals.

Entropy is minimal when the image consists of a single grey level and maximum when all grey levels

\begin{tabular}{|l|l|l|l|l|}
\hline 1 & 2 & 2 & 2 & 1 \\
\hline 0 & 1 & 0 & 2 & 2 \\
\hline 0 & 0 & 1 & 2 & 0 \\
\hline 2 & 1 & 0 & 1 & 1 \\
\hline 1 & 2 & 2 & 0 & 0 \\
\hline
\end{tabular}

Fig. 3 Three recurring grey level values 0,1 , and 2 are used in an arbitrary $5 \times 5$ pixel array to produce a simulated image. are equally likely to occur at adjacent pixel points. (25) ASM. The angular second moment is calculated as:

$$
\operatorname{ASM}=\sum_{i=0}^{N_{g}-1} \sum_{j=0}^{N_{g}-1} p(i, j)^{2}
$$

The angular second moment measures the degree of grey level homogeneity in the image and is closely but inversely related to entropy.

(26) LHOM. The local homogeneity is calculated as:

$$
\text { LHOM }=\sum_{i=0}^{N_{g}-1} \sum_{j=0}^{N_{g}-1} \frac{1}{1+(i-j)^{2}} p(i, j)
$$

It measures the similarity between neighbouring image points due to a weighting factor which gives more weight to neighbouring points with similar grey levels.

(27) INERT. The moment of inertia about the matrix diagonal is calculated as:

$$
\operatorname{INERT}=\sum_{i=0}^{N_{g}-1} \sum_{j=0}^{N_{g}-1}(i-j)^{2} p(i, j)
$$

This measures the dissimilarity of grey levels between adjacent points.

(28) CORREL. The correlation is calculated as:

$$
\text { CORREL }=\frac{\sum_{i=0}^{N_{y}-1} \sum_{j=0}^{N_{y}-1}\left(i-\mu_{x}\right)\left(j-\mu_{y}\right)}{\sigma_{x} \sigma_{y}} \text { where }
$$

$$
\begin{aligned}
& \mu_{x}=\sum_{i=0}^{N_{y}-1} i \sum_{j=0}^{N_{g}-1} p(i, j) \\
& \mu_{y}=\sum_{j=0}^{N_{y}-1} j \sum_{i=0}^{N_{y}-1} p(i, j) \\
& \sigma_{x}=\sum_{i=0}^{N_{y}-1}\left(i-\mu_{x}\right)^{2} \sum_{j=0}^{N_{y}-1} p(i, j) \\
& \sigma_{y}=\sum_{j=0}^{N_{y}-1}\left(j-\mu_{y}\right)^{2} \sum_{i=0}^{N_{y}-1} p(i, j)
\end{aligned}
$$

CORREL measures the degree to which the elements of the matrices are concentrated along the diagonal.

(d) Other texture measurements. Two additional texture features which have been found useful in computer analysis of chest radiographs were also calculated..$^{14}$ These are:

(29) MDIVERS. This measurement is based on the 
Table 1 Four symmetric grey level co-occurrence matrices derived from the image represented by Fig. 3. The numbers in the matrices indicate the estimated probability of changes in grey level values between adjacent pixels for horizontal, vertical, and the two diagonal directions. The matrix values are derived from the observed frequency of differences between neighbouring pixels

\begin{tabular}{ll}
$S_{0}=\frac{1}{40}\left|\begin{array}{lll}4 & 5 & 3 \\
5 & 2 & 5 \\
3 & 5 & 8\end{array}\right|$ & $S_{45}=\frac{1}{32}\left|\begin{array}{lll}2 & 3 & 5 \\
3 & 4 & 4 \\
5 & 4 & 2\end{array}\right|$ \\
$S_{40}=\frac{1}{40}\left|\begin{array}{lll}2 & 8 & 4 \\
8 & 0 & 5 \\
4 & 5 & 4\end{array}\right|$ & $S_{135}=\frac{1}{32}\left|\begin{array}{lll}6 & 2 & 3 \\
2 & 6 & 2 \\
3 & 2 & 6\end{array}\right|$ \\
\hline
\end{tabular}

diversity of the quantitised directions of the gradient vector within a local $3 \times 3$ window as it scans the nuclear area. The direction of the gradient vector is computed at each image element, which is labelled with an integer value from 1 to 8 according to which of the eight compass points it is most closely aligned to. In the nine elements of any $3 \times 3$ window there may be from one to eight differently labelled elements. The mean value of the number of differently labelled elements for all the $3 \times 3$ windows of the nuclear region is used as the texture measurement. This value will increase as adjacent gradient directions become more scattered.

(30) STDLAP. A measure of "spottiness" of the nucleus was made based on grey levels in a $4 \times 4$ local operator. If $C$ is the average grey value of the four central pixels and $S$ is the average value of the eight axially adjacent cells, then the value $\mathrm{C}-\mathrm{S}$ is computed for each $4 \times 4$ window. The "spottiness" of a region can be estimated from the standard deviation of this value over the region, provided that the "spots" are of the same order of magnitude as the size of the operator.

\section{Results}

The data file consisting of 30 variables for each nucleus was transferred to a DEC-20 computer and analysed using the BMDP statistical package.

The distributions of many of the measured variables are very asymmetric. Rather than attempt to apply normalising transformations, we chose to summarise the distribution of each by its median value and its range as defined by the difference between the 5th and 95th percentile values. Each biopsy was thus characterised by $\mathbf{3 0}$ median and 30 range variables.

In order to identify those variables which in isolation gave the best discrimination between nuclei from neoplastic and non-neoplastic biopsies, a nested random factors analysis of variance was computed for each of the 60 variables. There were 21 variables for which the estimated component of variance between the groups (normal $v$ neoplastic) was greater than the variance within the groups (Table 2). Expressing the variance ratio for 1 and 18 degrees of freedom in the usual way by a significance level is not meaningful because the variances of the variables in the neoplastic group were greater than those from the non-neoplastic group. An indication of the group differences is given by the difference between group means divided by the pooled standard deviation (d). The non-parametric KruskalWallis test ${ }^{17}$ was used to compute a significance level for each of these variables.

The features which most effectively distinguished between the two groups were shape measurements. It is seen from Table 2 that the range of a shape measurement within biopsy samples is a better discriminant than the corresponding median value.

Five of the 21 variables were derived from the nuclear texture. The most effective of these are the median values of three measurements derived from the co-occurrence matrices. Surprisingly, the size of the nuclei played a less important role, but the range of nuclear sizes within a biopsy did show a minor discriminatory effect.

Many of the variables measure similar characteris-

Table 2 Eleven median and 10 range features for which the estimated component of variance between the normal and neoplastic biopsies was greater than the variance between biopsy samples. The distance between means divided by the pooled standard deviation $(d)$ is an indication of the feature discriminatory power. The significance level $p$ is derived from the non-parametric Kruskal-Wallis test statistic

\begin{tabular}{|c|c|c|c|c|c|}
\hline Median feature & $d$ & $p$ & Range feature & $d$ & $p$ \\
\hline $\begin{array}{l}\text { PERIM } \\
\text { P2A } \\
\text { AVCURV } \\
\text { AVDIS } \\
\text { STDIS } \\
\text { MINDIS } \\
\text { MAXDIS } \\
\text { ARANGE } \\
\text { CORREL } \\
\text { LHOM } \\
\text { INERT }\end{array}$ & $\begin{array}{l}1 \cdot 78 \\
1 \cdot 78 \\
1 \cdot 72 \\
2 \cdot 42 \\
2 \cdot 35 \\
2 \cdot 04 \\
1.81 \\
1 \cdot 50 \\
2 \cdot 15 \\
1.98 \\
2 \cdot 23\end{array}$ & $\begin{array}{l}0 \cdot 0005 \\
0 \cdot 0008 \\
0 \cdot 001 \\
0 \cdot 0008 \\
0 \cdot 001 \\
0 \cdot 001 \\
0 \cdot 002 \\
0 \cdot 005 \\
0 \cdot 0007 \\
0 \cdot 001 \\
0 \cdot 0007\end{array}$ & $\begin{array}{l}\text { SIZE } \\
\text { PERIM } \\
\text { P2A } \\
\text { AVCURV } \\
\text { SSQCURV } \\
\text { AVDIS } \\
\text { STDIS } \\
\text { MINDIS } \\
\text { MAXDIS } \\
\text { MGRAD }\end{array}$ & $\begin{array}{l}1 \cdot 66 \\
3 \cdot 20 \\
2 \cdot 96 \\
1 \cdot 99 \\
1 \cdot 61 \\
2 \cdot 60 \\
3 \cdot 60 \\
2 \cdot 99 \\
2 \cdot 81 \\
1 \cdot 71\end{array}$ & $\begin{array}{l}0.002 \\
0.0002 \\
0 \cdot 0002 \\
0 \cdot 0002 \\
0.0007 \\
0 \cdot 0002 \\
0 \cdot 0002 \\
0 \cdot 0002 \\
0 \cdot 0002 \\
0.008\end{array}$ \\
\hline
\end{tabular}


Table 3 Correlations between five features derived from the co-occurrence matrices. There, are strong correlations between ENT and ASM and between LHOM, INERT, and CORREL

\begin{tabular}{llllc}
\hline & ASM & LHOM & INERT & CORREL \\
\hline ENT & 0.92 & 0.65 & -0.55 & 0.56 \\
ASM & & 0.64 & -0.48 & 0.50 \\
LHOM & & & -0.97 & 0.94 \\
INERT & & & & -0.93 \\
\hline
\end{tabular}

tics and are therefore correlated. For example, the mean of the ranked correlation coefficients for the five textural measurements derived from the cooccurrence matrices are shown in Table 3 , from which it is apparent that they fall into two groups. It is possible that ASM and ENTROPY are measuring a different nuclear characteristic from LHOM, INERT, and CORREL.

A stepwise linear discriminant program was used to select a combination of variables which best separated the two groups of biopsies. Because of the small number of specimens (20 biopsies), the program was allowed to select only the two most important variables.

The best discriminating variable is the one with the largest $d$ value (Table 2). This is a shape characteristic - the range of STDIS - which measures the range over 30 cells of the standard deviation of the distance of the nuclear boundary from its centre of gravity. This shape feature completely separates the two groups of biopsies (Fig. 4).

The second most important variable which was selected by the program after allowing for the between variable correlations was the median value of the texture measurement INERT. On its own this did not completely separate the two groups of biopsies (Fig. 5). The between group separation using a combination of these two variables is shown in Fig. 6.

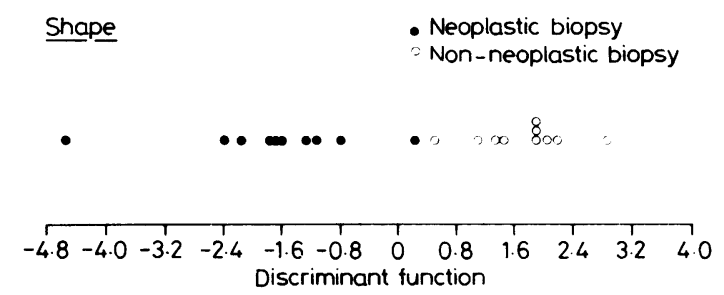

Fig. 4 Separation of non-neoplastic and neoplastic liver biopsies based on the distance of the nuclear boundary to its centre of gravity in groups of 30 cells.

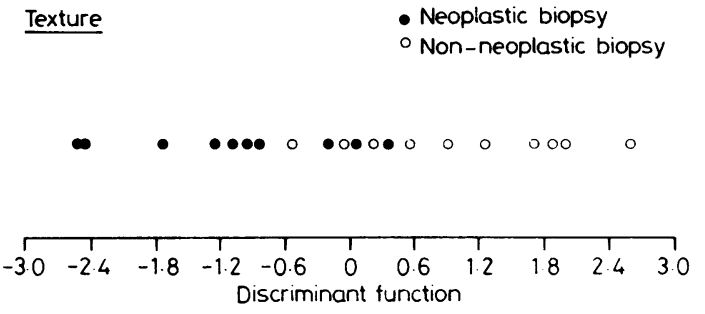

Fig. 5 Separation of non-neoplastic and neoplastic liver biopsies on the texture measurement INERT in groups of 30 cells.

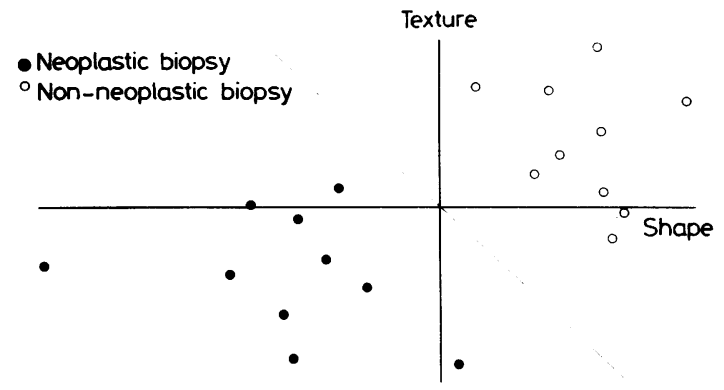

Fig. 6 Separation of the two groups of biopsies using a linear discriminant based on nuclear shape and texture.

\section{Discussion}

Histopathologists base their diagnosis and grading of malignancies on many features: tissue architecture, cytoplasmic appearances, and changes in the nuclei. Their diagnoses are based on a considered summation of many factors and not on any single one. Nevertheless, it is important to try to separate the features and to verify their importance by objective measurement.

Nuclear features such as size and changes in shape are important. They may be assessed by simple measurements and by shape factors such as deviations from circularity and changes in curvature functions of the nuclear boundary. ${ }^{8}$

It is more difficult to measure the state of the nucleus described subjectively as "coarse," "spotty," "dispersed," or "clumped." Changes underlying such nuclear appearances may reflect quantitative alterations in the nuclear content of DNA, but they also represent functional states of the nucleus with dispersion or aggregation of the nuclear material. Nicolini et $a l^{15}$ have reported that the texture of W1-38 fibroblast nuclei show different textures related to stages in the cell cycle, though the DNA content remains constant.

Changes in the appearance of the nucleus are 
measured by texture analysis. The texture of an image may be defined as regional patterns of grey level density distributions in an image which are usually locally recurrent. ${ }^{16}$ Such techniques have been widely used in meteorology and in Landsat descriptions of terrain, ${ }^{12}{ }^{13}$ but their use in biology has been less common, partly because of the complexity of the equipment to make such measurements. Lipkin and Lipkin, ${ }^{16}$ however, have used texture to assess nuclear maturation in granulocytes, and Ștenkvist et $^{\mathrm{al}} \mathbf{l}^{7}$ have characterised breast cancer cell populations, partly on the basis of texture. Computer assisted microscopy is now relatively inexpensive and textural analysis is a potential tool for pathologists.

In this report we have expanded our previous study on nuclear size and shape as discriminants of hepatoma cells by measurement of additional shape factors and measurement of the nuclear texture. The grouped values of nuclear features obtained from the measurements on 30 cells in each biopsy show that when malignant samples are compared with non-neoplastic samples there are many differences between the two groups in size, shape, and texture measurements. The best separation is given by a feature which is a measurement of the range of values within a biopsy of a function derived from the distance of the nuclear boundary from its centre of gravity. This feature is closely related to one derived from the curvature function which was previously identified as a good discriminator of neoplastic cells. ${ }^{8}$ A random selection of cells from neoplastic biopsies is likely to include a proportion of nonneoplastic cells in the sample. This dilution of the neoplastic cells is the most obvious reason for the range of shape variables to play a greater discriminatory role than the median values.

The median value of the measurement INERT was the second best discriminant but this did not completely separate the two groups of biopsies

The range of nuclear sizes within a biopsy did, in isolation, show some group separating effect. When the two factors of shape and texture were analysed by the linear discriminant techniques, however, the size effect disappeared.

It is of interest, but disappointing, that those measurements of texture which reflect aggregation of stained nuclear material near to the nuclear membrane did not appear to be of value in discriminating either individual cells or biopsies. The sample size in this analysis was small ( 20 biopsies) and it is likely that with larger samples other features may have a discriminant role.

This study has shown that objective measurements may be made by computer assisted microscopy on liver cell nuclei in histological sections pre- pared by standard techniques. Measurements of size, shape, and texture show significant differences between neoplastic and non-neoplastic biopsies. The liver was chosen for this study as an ideal subject for computer analysis with widely separated nuclei which facilitated the development of automatic nuclear detection. The sections examined were stained by haematoxylin and eosin in order to assess the images normally seen by pathologists. Automatic nuclear detection depends on sophisticated programs, but nuclear definition may be enhanced by alternative embedding and staining techniques. But these alternatives may alter the information obtained. These aspects are under study.

It is important to emphasise that objective measurements of nuclei by computer assisted microscopy cannot replace the diagnostic process used by the histopathologist. This is a subtle process of assessing tissue architecture and cellular context as well as the structure of nuclei and cytoplasm and is underpinned by the experience of the pathologist. Moreover, the criteria used may not be the same in different tissues. Nevertheless, such cellular measurements as described are valuable in that they lead to a more critical appraisal of what the pathologist actually sees. Moreover, they may be of importance as an adjunct to diagnosis in providing some objective data for tumour grading where human assessment is notoriously inconsistent and variable.

We thank Mrs Sheila Dandy for her help in this work and Mr CE Rossiter and Dr ID Hill for statistical help and advice.

\section{References}

Baak JPA, Diegenbach PP. Quantitative nuclear image analysis: differentiation between normal, hyperplastic and malignant appearing uterine glands in a paraffin section 1. Elementary features for differentiation. European Journal of Obstetrics, Gynaecology and Reproductive Biology 1977;5:33-42.

${ }^{2}$ Abbot CR, Blewitt RW, Bird CC. Quantitative analysis of nonHodgkin's lymphoma. J Clin Pathol 1982;35:135-8.

${ }^{3}$ Crocker J, Curran RC. A study of nuclear diameters in lymph node imprints using the Zeiss Videomat. J Clin Pathol 1979;32:670-4.

4 Boon ME, Kurver PJH, Baak JPA, Thompson HT. The application of morphology in gastric cytological diagnosis. Virchows Arch A (Pathol Anat) 1981;393:159-64.

${ }^{5}$ Stinson SF, Lilga JC, Reese DH, Friedman RD, Sporn MB. Quantification with an automated image analyser of nuclear cytoplasmic changes induced by hydrocortisone in bladder epithelium. Cancer Res 1977;37:1428-31.

- Ooms ECM, Essed E, Veldhvizen RW, Kurver PJH, Boon ME. The prognostic significance of morphology in $\mathrm{T} 1$ bladder tumors. Histopathology 1981;5:311-8.

' Stenkvist B, Westman-Naer S, Holmquist J, et al. Computerised nuclear morphometry as an objective method for characterising human cancer cell populations. Cancer Res 1978; 38:4688-97. 
8 Jagoe R, Sowter C, Dandy S, Slavin G. Morphometric study of liver cell nuclei in hepatomas using an interactive computer technique: 1) Nuclear size and shape. J Clin Pathol 1982;35:1057-62.

${ }^{9}$ Haralick RM. Automatic remote sensor image processing. In: Rosenfeld A, ed. Topics in applied physics Vol II: Digital picture analysis. New York: Springer-Verlag, 1976.

${ }^{10}$ Eccles MJ, McQueen PC, Rosen D. Analysis of the boundaries of planar objects. Pattern Recognition 1977;9:31-41.

"Thomas JO. Texture analysis in image processing. In: Thomas JO, Davey PG, eds. Texture analysis. Proceedings of British Pattern Recognition Association and Remote Sensing Society. Oxford: Physics Photographic Unit, Oxford University, 1977.

${ }^{12}$ Harris R. Automatic analysis of meteorological satellite imagery. In: Thomas JO, Davey PG, eds. Texture analysis. Proceedings of British Pattern Recognition Association and Remote Sensing Society. Oxford: Physics Photographic Unit, Oxford University, 1977.

${ }^{i 3}$ Weszka JS, Dyer CR, Rosenfeld A. A comparative study of tex- ture measures for terrain classification. IEEE Transactions on Systems, Man and Cybernetics 1976;4:269-85.

14 Jagoe JR. Measurement of pneumoconiosis in chest films by computer. In: Gardner WE, ed. Machine-aided image analysis. Institute of Physics Conference Series no 44. Bristol: Institute of Physics, 1978.

is Nicolini C, Kendall F, Basergen R, Dessaive C, Clarkson B, Fried J. The G0-G1 transition of W138 cells. II. Geometric and densitometric texture analysis. Experimental Cell Researck 1977; 106: 1 19-25.

${ }^{16}$ Lipkin BS, Lipkin LE. Textural parameters related to nuclear maturation in the granulocytic leucocytic series. J Histochem Cytochem 1974; 22:583-94.

${ }^{17}$ Kruskal WH, Wallis WA. Use of ranks in one-criterion variance analysis. J Am Statist Assoc 1952;47:583-621.

Requests for reprints to: Mr R Jagoe, Department of Computing, Northwich Park Hospital, Harrow, Middlesex HA1 3UJ, England. 